\title{
Doublonlike Excitations and Their Phononic Coupling in a Mott Charge-Density-Wave System
}

\author{
C. J. Butlerø, ${ }^{1, *}$ M. Yoshida ${ }^{1}{ }^{1}$ T. Hanaguri ${ }^{1, \dagger},{ }^{\dagger}$ and Y. Iwasa ${ }^{1,2}$ \\ ${ }^{1}$ RIKEN Center for Emergent Matter Science, 2-1 Hirosawa, Wako, Saitama 351-0198, Japan \\ ${ }^{2}$ Quantum-Phase Electronics Center and Department of Applied Physics, The University of Tokyo, \\ 7-3-1 Hongo, Bunkyo-ku, Tokyo 113-8656, Japan
}

(Received 29 September 2020; revised 1 February 2021; accepted 9 February 2021; published 24 March 2021)

\begin{abstract}
Electron-phonon-driven charge-density waves can in some circumstances allow electronic correlations to become predominant, driving a system into a Mott insulating state. New insight into both the Mott state and preceding charge-density wave may result from observations of the coupled dynamics of their underlying degrees of freedom. Here, tunneling injection of single electrons into the upper Hubbard band of the Mott charge-density-wave material $1 T-\mathrm{TaS}_{2}$ reveals extraordinarily narrow electronic excitations which couple to amplitude mode phonons associated with the charge-density wave's periodic lattice distortion. This gives a vivid microscopic view of the interplay between excitations of the Mott state and the lattice dynamics of its charge-density wave precursor.
\end{abstract}

DOI: 10.1103/PhysRevX.11.011059

Subject Areas: Condensed Matter Physics, Strongly Correlated Materials

\section{INTRODUCTION}

Coupling between electronic and lattice degrees of freedom underpins many intriguing and useful phenomena in condensed matter systems, such as charge-density waves (CDWs) and the pair-binding mechanism in conventional superconductivity [1-4]. The layered transition metal dichalcogenide $1 T-\mathrm{TaS}_{2}$ is a material whose rich electronic phase diagram and ground state electronic properties are shaped principally by complex electronphonon (e-ph) interactions. At low temperature, $e$-ph interactions drive a commensurate CDW described by a $\sqrt{13} \times \sqrt{13} R 13.9^{\circ}$ superlattice. Figure 1 (a) shows a typical scanning tunneling microscopy (STM) image acquired in this phase, at a temperature of $1.5 \mathrm{~K}$. This CDW can equivalently be described as a lattice of polaronic star-of-David (SD) clusters each encompassing 13 Ta ions [1] and formed from the symmetrical contraction of 12 of the Ta ions toward a central one, as depicted in Fig. 1(b).

The most widely adopted understanding of the ground state in $1 T-\mathrm{TaS}_{2}$ is that the $\mathrm{CDW}$ reduces the electronic bandwidth below the threshold for a Mott

\footnotetext{
*christopher.butler@ riken.jp

†hanaguri@ riken.jp
}

Published by the American Physical Society under the terms of the Creative Commons Attribution 4.0 International license. Further distribution of this work must maintain attribution to the author(s) and the published article's title, journal citation, and DOI. transition into an insulating state [6,7]. Nevertheless, much is yet to be clarified about the driving mechanisms behind both the CDW itself and the Mott state, and about the detailed interplay between $e$-ph interactions and electronic correlations. Time-resolved photoexcitation measurements have provided fresh insight by investigating the system's dynamics, from excitation of the CDW amplitude mode [8] and doublons [characteristic excitations of the upper Hubbard band (UHB) of a Mott insulator] in the weak-perturbation regime $[9,10]$ to timing the melting of both the Mott state and the CDW toward metallic and other phases under highintensity light [11-14].

Here we report on a spectroscopic signature of the dynamic coupling between electronic and lattice degrees of freedom in $1 T-\mathrm{TaS}_{2}$, with the electronic perturbation provided not by photoexcitation but instead by local tunneling injection of single electrons using scanning tunneling microscopy. Specifically, injection of electrons into unusual and previously unobserved narrow states found at the UHB onset likely excites amplitude mode phonons associated with the CDW lattice distortion. The narrow states themselves may evidence either long-lived coherent quasiparticles existing at the UHB edge or possible polaronic bound states formed upon injection. These microscopic observations complement the ensemble-averaged ultrafast optical and time-resolved photoemission spectroscopy measurements and also provide a reference point for theories modeling exotic excitations of Mott-Hubbard and related systems. 
(a)

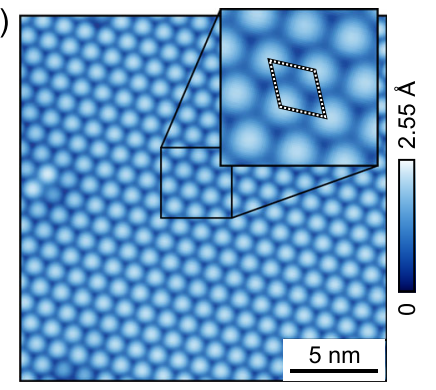

(b)

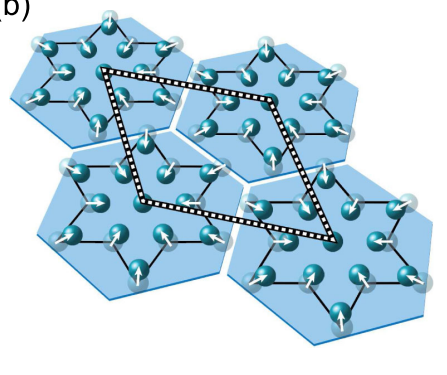

(c)

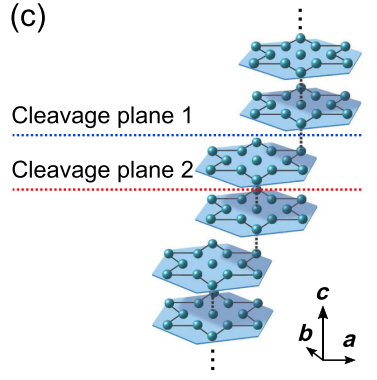

(d)

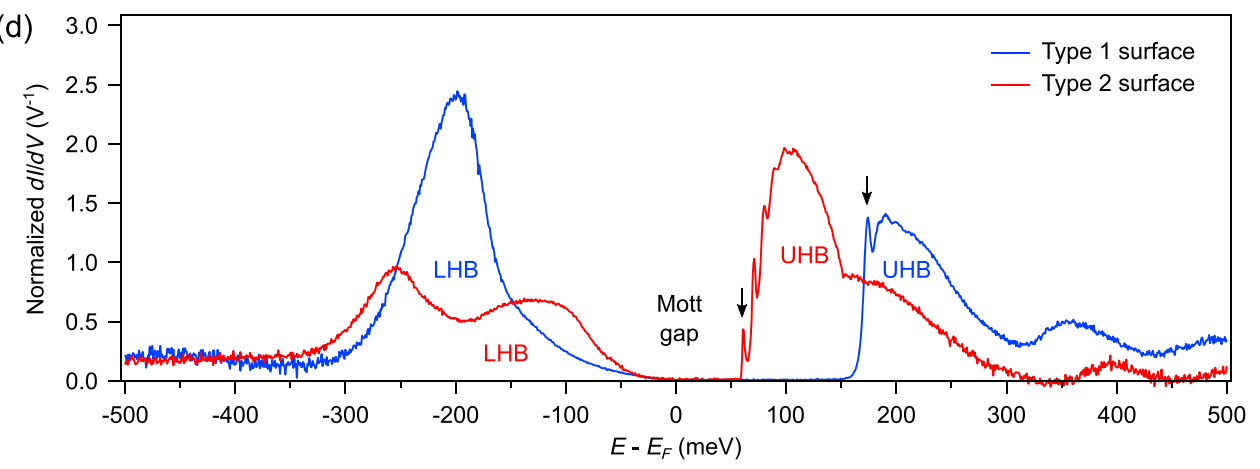

FIG. 1. Spectroscopically distinct surface terminations of the three-dimensional charge order in $1 T-\mathrm{TaS}_{2}$. (a) A typical constantcurrent topograph $(V=250 \mathrm{mV}, I=250 \mathrm{pA})$, acquired at a type 1 surface. (b) A depiction of the periodic lattice distortion forming SD clusters with $13 \mathrm{Ta}$ atoms each (sulfur ions are neglected). A surface-projected supercell of the CDW distortion is shown with a black and white dashed line, corresponding to the cell shown in the inset of (a). (c) The alternating interlayer stacking of the SD clusters, showing the two inequivalent cleavage planes 1 and 2. (d) High-resolution $(d I / d V)(V)$ curves acquired at two types of surface. Recent STM investigations allow the identification of each spectrum with the type 1 and 2 surfaces formed by cleavage through the planes 1 and 2 in (c) [5]. The newly observed peaks at each surface are indicated with arrows. For ease of comparison, the curves are normalized by dividing by the current value at $+500 \mathrm{mV}$ for each.

\section{RESULTS}

High-energy-resolution tunneling conductance $(d I / d V)$ spectra were acquired at two distinct surface terminations of the three-dimensional CDW pattern. The observation of two surfaces is relevant as follows: The status of $1 T-\mathrm{TaS}_{2}$ as a Mott insulator was recently challenged $[15,16]$ with the suggestion that staggered interlayer stacking [17-19] could result in a simple band insulator through Peierls-like interlayer dimerization. There is now mounting evidence from state-of-the-art x-ray diffraction [20], high- and lowenergy electron diffraction [21,22], and STM experiments [5] that such an alternating stacking pattern is indeed realized in the low-temperature phase, and the band insulating picture has attracted some support [23]. Nevertheless, we recognize that Peierls-like dimerization and an electron-correlation-driven insulating state are not mutually exclusive [24], and behaviors consistent with a correlation-driven insulator continue to be reported [5,10,25]. As depicted in Fig. 1(c), the stacking pattern features two inequivalent cleavage planes, labeled as planes 1 and 2, forming dimerized or undimerized surfaces which yield distinct tunneling spectra of type 1 or 2 [blue and red curves in Fig. 1(d)], respectively. As we suggested recently, if the interlayer stacking pattern is truncated at a surface (namely, of type 2) leaving an undimerized layer, the persistence of a gap in absence of dimerization suggests electronic correlations as the determining mechanism [5]. Hence, in this work we adopt the viewpoint that the major spectroscopic features described below can be interpreted in the Mott-Hubbard picture.

Particularly noteworthy in these spectra are the exceptionally narrow peaks appearing at the onset of unoccupied states at each surface, marked with black arrows in Fig. 1(d). These features have a width of only $3-5 \mathrm{meV}$, or a few percent of the total bandwidth of the UHB. This observation is enabled by the high energy resolution afforded by a lockin technique using a bias modulation $V_{\text {mod }}=1 \mathrm{mV}$ or less, with a suitable sampling interval, and would be missed in measurements using the more typical modulation and sampling interval of $\sim 10 \mathrm{mV}$ or more. We note that such features appear to be absent in the occupied states, and the reasons for this are discussed below.

Spatially resolved tunneling spectroscopy measurements shown in Fig. 2 give a more complete overview of the behavior seen at the UHB edge at each surface. Figures 2(a) and 2(b) show topographs acquired simultaneously with spectroscopy measurements, and in these the type 1 and 2 surfaces appear nearly indistinguishable. Figures 2(c) and 2(d) show spectroscopic line cuts along each of the arrows 
(a)

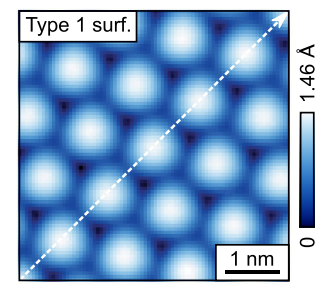

(c) 2

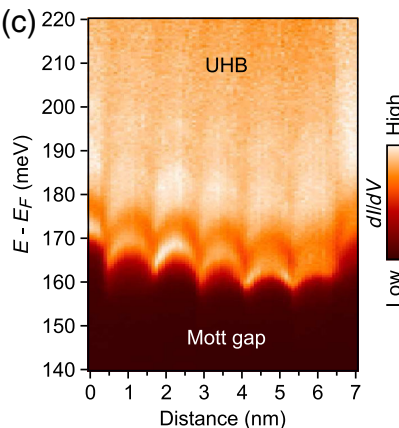

(b)

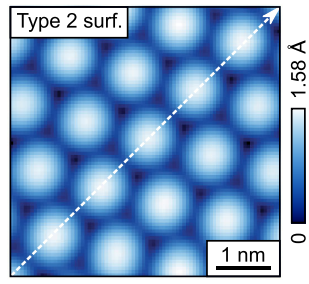

(d)

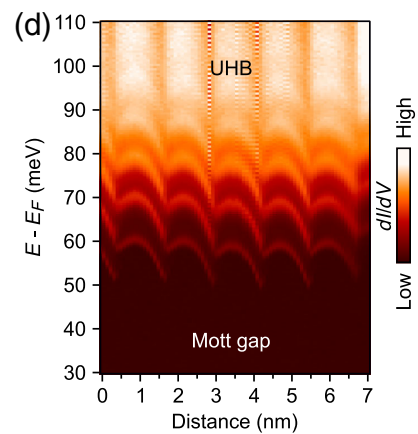

FIG. 2. Spectroscopic imaging at the UHB onset at type 1 and 2 surfaces. (a),(b) Constant-current topographs of each surface (setpoints $\quad V=0.25 \mathrm{~V}, \quad I=1 \mathrm{nA}, \quad$ and $\quad V=0.11 \mathrm{~V}$, $I=0.44 \mathrm{nA}$, respectively). (c),(d) Spectroscopic line cuts taken along the white dashed lines shown in (a) and (b). Note that each energy range is chosen for suitability to each UHB onset energy. From here onward, this work focuses on the features observed at the type 2 surface.

shown in Figs. 2(a) and 2(b). Here the narrow peaks appear as arclike features, with at least one arc (and five arcs) spanning each cluster for the type 1 (type 2) surface. This arclike shape is consistent with a distortion by tip-induced effects of an intrinsically flat, tilelike structure. Despite this, the energy spacing between arcs appears rigid and position independent. In the Supplemental Material, we further discuss the tip-induced effects, the underlying spatial distribution of the features as it relates to Mott localization, and also the differences between the type 1 and 2 spectra [26].

Below we examine the series of peaks at the type 2 surface in particular, following the hypothesis that they result from replication of the zeroth peak by $e$-ph interactions. An example of a typical spectrum acquired on a cluster at the type 2 surface is analyzed, as shown in Fig. 3. Similar analyses of spectra acquired in different sample locations are shown in the Supplemental Material [26].

Given some sharp spectroscopic feature, the relative intensities of its $e$-ph replicas are governed according to the Franck-Condon principle, which is commonly used to describe absorption line shapes corresponding to an electronic excitation-for example, a photoexcited vertical transition-coupled to a single optical phonon or vibron mode [27]. The Franck-Condon principle has also been used to interpret STM observations of the vibronic properties of organic molecules resting on an insulating substrate [28-30]. There, injection of an electron into the lowest
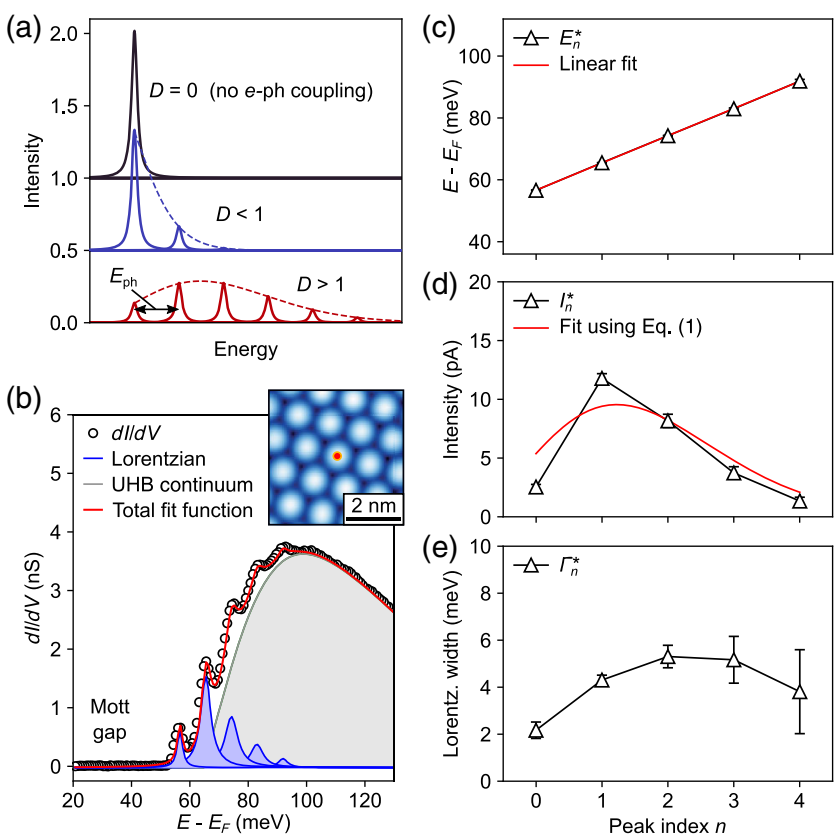

FIG. 3. Analysis of $e$-ph replica peaks at the type 2 surface. (a) Idealized line shapes described by Eq. (2) for various values of $D$. The dashed lines show envelope functions for the relative peak intensities according to the Franck-Condon principle [Eq. (1)]. Plots are vertically offset for clarity. (b) Fitting, using Eq. (3), to the conductance spectrum of unoccupied states at the type 2 surface, acquired at the point shown in the inset. (c) Fitted energies for the peaks in (b), with a linear fit giving an average spacing of $8.8 \mathrm{meV}$. (d) Fitted peak intensities (total area under each Lorentzian curve), with further fitting using Eq. (1) yielding the Huang-Rhys parameter $D=1.75$. (e) Fitted Lorentzian broadening parameters for each peak.

unoccupied molecular orbital (LUMO) formed a long-lived transient charged state, exciting molecular vibron modes which produced a series of replicas of the LUMO. Drawing an analogy with this, and regarding the SD cluster here as a "pseudomolecule," the sharp state near the onset of the UHB can be thought of as corresponding to the LUMO. Instead of vibron modes, a charged state of the SD cluster may couple to lattice phonons. Generally, $e$-ph replicas are discernible only when the width of the spectroscopic feature is small compared to the phonon energy. Where in previous cases, such as doped diamond [27] or the aforementioned molecular adsorbate, phonon or vibron energies were sufficient that replicas were seen for a broadband or molecular orbital, here only the narrow feature at the UHB edge is discernibly replicated. We return to the possible origin of the narrow state itself below.

The Franck-Condon scheme gives the relative intensities of replica peaks resulting from the emission of $n$ phonons. The intensity of the $n$th peak is

$$
I_{n}=A e^{-D} \frac{1}{n !} D^{n},
$$


where $A$ is a scaling coefficient common to the whole series and $D$ is the Huang-Rhys parameter characterizing the strength of the electron-ion coupling [31].

The expected line shape for a conductance spectrum $g(V) \equiv(d I / d V)(V)$ can be expressed as a Franck-Condon progression of peaks, each convolved with a suitable broadening function, on a background function which in this case represents the UHB continuum. This can be written as

$$
\begin{aligned}
g(V) \propto & \sum_{n=0}^{\infty}\left[e^{-D} \frac{1}{n !} D^{n} \int \frac{\Gamma_{n} / 2}{\left(q_{e} V\right)^{2}+\left(\Gamma_{n} / 2\right)^{2}}\right. \\
& \left.\times \delta\left(q_{e} V-E_{0}-n E_{\mathrm{ph}}\right) d V\right]+\mathrm{UHB} \text { continuum, }
\end{aligned}
$$

where $n \in\{0,1,2, \ldots\}, \Gamma_{n}$ are peak-specific broadening parameters, $q_{e}$ is the electron charge, $E_{0}$ is the energy of the underlying narrow electronic state, and $E_{\mathrm{ph}}$ is the phonon energy. The resulting ideal line shapes given by Eq. (2), ignoring the UHB continuum, are depicted for a few values of $D$ in Fig. 3(a).

In practice, in order to perform fitting to the conductance spectra and extract the key quantities $E_{\mathrm{ph}}, D$, and $\Gamma_{n}$, a modified expression was used. First, $n$ was only allowed to run up to a value of 4 (for a total of five peaks), and in order to achieve convergence the intensities, energies, and widths of each of the individual peaks were treated as free parameters (marked with asterisks). Finally, convolution with the semicircular resolution function $\lambda(V)$ associated with the lock-in technique was taken into account:

$$
\begin{aligned}
g(V) \approx & \sum_{n=0}^{4}\left[\frac{I_{n}^{*} \Gamma_{n}^{*} / 2}{\left(q_{e} V-E_{n}^{*}\right)^{2}+\left(\Gamma_{n}^{*} / 2\right)^{2}} * \lambda(V)\right] \\
& + \text { UHB continuum, }
\end{aligned}
$$

where

$$
\lambda(V)= \begin{cases}\frac{1}{\pi V_{\text {mod }}} \sqrt{1-\left(\frac{V}{V_{\text {mod }}}\right)^{2}} & \text { if }|V| \leq V_{\text {mod }} \\ 0 & \text { otherwise. }\end{cases}
$$

The result of fitting is shown in Fig. 3(b). A phenomenologically driven choice was made for a line shape to represent the UHB continuum, namely the upper half of a skew-normal function. Error bars shown in each plot correspond to the square roots of the diagonal elements of the estimated covariance matrix obtained alongside the optimized values.

The resulting energies $E_{n}^{*}$ are plotted in Fig. 3(c), along with a linear fit that finally yields the average spacing $E_{\mathrm{ph}}=8.8 \mathrm{meV}$. Fitting to curves acquired in multiple
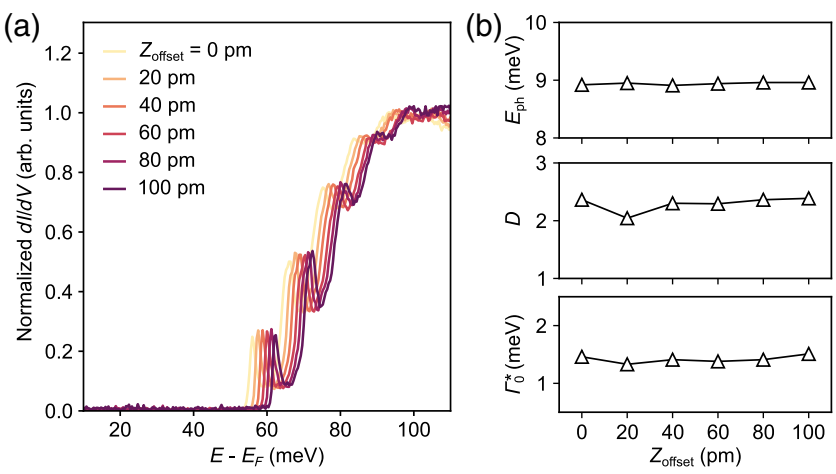

FIG. 4. Insensitivity of Franck-Condon series to tip-sample separation. (a) Conductance curves collected at varying STM tip height. After stabilizing the tip at the height $z_{\text {setpoint }}$ (at $V=0.11 \mathrm{~V}, I=0.44 \mathrm{nA}$ ), the tip height was altered by an amount $z_{\text {offset }}$ before measuring the conductance spectrum. Here the curves are normalized for ease of comparison, using the value of $d I / d V$ at an energy $40 \mathrm{meV}$ above that of the lowest-lying peak. (b) Results of the fitting with the function in Eq. (3). The parameters $E_{\mathrm{ph}}, D$, and $\Gamma_{0}^{*}$ are insensitive to the tip height.

locations on the sample shows that values of $E_{\mathrm{ph}} \approx$ $9 \mathrm{meV}$ are typical.

The fitted peak intensities $I_{n}^{*}$ are plotted in Fig. 3(d), with the subsequently fitted curve for the Franck-Condon weights [from Eq. (1)] giving $D=1.75$. From the discussion which follows, we can infer only that this value is below the threshold for formation of a self-trapped polaron [32]. The broadening of each peak, plotted in Fig. 3(e), shows the expected tendency that $\Gamma_{n}^{*}$ increases with higher energy, suggesting increased damping further into the UHB continuum. Here the most significant value is the broadening of the lowest-lying peak, $\Gamma_{0}^{*}=2.17 \mathrm{meV}$, to which we return below.

Figure 4 shows conductance spectra, and the result of fitting using Eq. (3), obtained on a different type 2 surface in a similar way to those shown in Fig. 3, for various tipsample separations, $z=z_{\text {setpoint }}+z_{\text {offset }}$. Varying $z$ has the effect of changing the electric field between the tip and sample (which is likely the source of the small energy shift with varying $z$ ), as well as changing the current by about one order of magnitude per $100 \mathrm{pm}$. The current is inversely proportional to the average time interval between tunneling events (see the discussion below). We see that $E_{\mathrm{ph}}, D$, and $\Gamma_{0}^{*}$ do not show any systematic variation with $z$. This insensitivity is expected, as the phonon energy, electron-phonon coupling strength, and peak width should be intrinsic properties of the sample.

\section{DISCUSSION}

In order to identify the apparent phonon mode observed above, its energy can be compared against previously reported Raman, time-resolved photoelectron, and ultrafast optical spectroscopy measurements on the low-temperature 
(a)

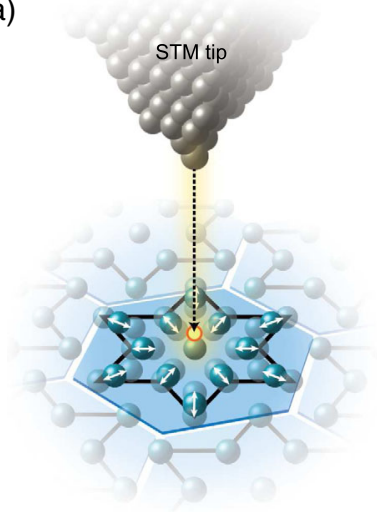

(b)

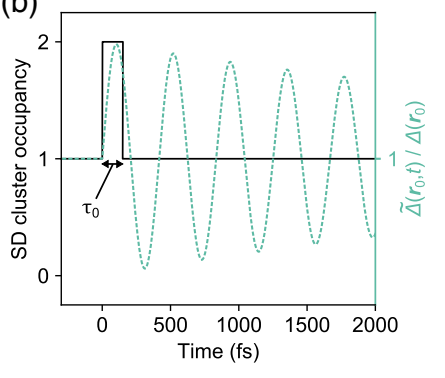

FIG. 5. Excitation of CDW amplitude mode by a transient doublonlike excitation. (a) A depiction of the excited amplitude mode in the SD cluster. (The Ta ionic displacements are greatly exaggerated.) (b) A qualitative plot of the SD cluster occupancy as a function of time, and the resulting local fluctuation of the order parameter.

phase. The energy $E_{\mathrm{ph}} \approx 9 \mathrm{meV}$ corresponds to a Raman shift of about $71 \mathrm{~cm}^{-1}$, and a strong Raman-active mode of this frequency has been observed, alongside other nearby modes [33,34], though the phonon species were not identified. Time-resolved pump-probe spectroscopy experiments have observed photoinduced excitations coupling to the amplitude mode of the $1 T-\mathrm{TaS}_{2} \mathrm{CDW}$, at a frequency $f_{\mathrm{AM}} \approx 2.4 \mathrm{THz}(9.9 \mathrm{meV})[8,9,35,36]$. Some reports have also described a mode at a frequency of $f \approx 2.1 \mathrm{THz}$ $(8.6 \mathrm{meV})[37,38]$, consistent with one speculated as either an $E_{g}$ or $A_{1 g}$ mode [34]. Like the amplitude mode, it appears only upon cooling into the commensurate CDW phase $[34,39]$.

From symmetry, it is reasonable that injection of an electron into the orbital localized at the SD center selectively excites the amplitude mode: The ionic arrangement in the host SD cluster responds to this excess negative charge by further symmetrically contracting toward the cluster center, setting the amplitude mode in motion. This scenario is depicted in Fig. 5(a). Though the observed phonon energy is also close to that of the previously reported $E_{g}$ or $A_{1 g}$ mode, it is not clear how a phonon of such symmetry can be excited by injection of an electron into the centrally localized orbital of the cluster.

Assuming that the orbital is fairly well localized to each cluster, the injected electron should be expected to dwell there for a finite time, before hopping away to neighboring clusters. This lifetime can be roughly estimated by considering the width of the zeroth peak in the series $\Gamma_{0}^{*}$ and using the uncertainty relation: $\Gamma_{0}^{*} \tau_{0} \geq \hbar / 2$. This yields a lifetime of $\tau_{0} \approx 152$ fs for the spectrum shown in Fig. 3(b). (For comparison, the timescale of electron hopping and screening in generic noninteracting systems is of the order $1 \mathrm{fs}$.) This long lifetime may attest to strong correlations in the half filled background, since in the Fermi-Hubbard

model the lifetime of a charged excitation residing in the UHB (a doublon) scales exponentially with the Mottness ratio (the ratio of the energy cost of double occupancy $U$ to the bandwidth $W$ ) [40].

The timescale for one period of the amplitude mode oscillation is $\left(1 / f_{\text {AM }}\right) \approx 417$ fs. Figure 5 (b) qualitatively shows the double occupancy of the SD cluster as a transient perturbation which induces a response of the lattice. Taking the periodic lattice distortion (or equivalently the ionic charge modulation) as the order parameter, and writing it as $\psi(\boldsymbol{Q})=\Delta(\boldsymbol{Q}) e^{i \phi}$, after a Fourier transform to real space the amplitude can be sampled at the SD cluster center $\boldsymbol{r}_{0}$ and labeled as $\Delta\left(\boldsymbol{r}_{0}\right)$. This is the equilibrium about which the amplitude mode will oscillate ( $\phi$ is fixed). Introducing time dependence with $\psi(\boldsymbol{r}, t)$, the modulated amplitude at $\boldsymbol{r}_{0}$ is $\tilde{\Delta}\left(\boldsymbol{r}_{0}, t\right)$. The local modulation $\tilde{\Delta}\left(\boldsymbol{r}_{0}, t\right) / \Delta\left(\boldsymbol{r}_{0}\right)$ is qualitatively plotted as a function of time in Fig. 5(b) (green dashed curve) along with the cluster occupancy. Previously the amplitude mode has been observed to persist for a timescale on the order $\sim 10 \mathrm{ps}$ [36]. Note that all the timescales depicted in Fig. 5(b), including the attenuation over time of the amplitude mode, are short compared to the average interval between tunneling of electrons from the tip to the sample. This time interval is $\sim 16 \mathrm{~ns}$ at the current of $\sim 10 \mathrm{pA}$ that was typically recorded just above the zeroth peak for the setpoint parameters used for the measurements described in Figs. 2 and 3. As the system recovers to equilibrium in a time much shorter than this interval, the phenomena described can be considered as the response of the system upon injection of a single electron.

From Fig. 5 it is reasonable to intuit that the lifetime $\tau_{0} \approx 152$ fs is of a suitable scale to trigger an oscillation of period $\approx 417 \mathrm{fs}$, since it covers most of the oscillation's first upward swing. Corollary to this, if $\tau_{0}$ tended toward zero, the coupling to the oscillation would diminish, i.e., $D$ would tend to zero. In the opposite limit, $\tau_{0} \rightarrow \infty$ (selftrapped polaron), the amplitude mode excitation would still be expected, oscillating around a new equilibrium lattice distortion.

We now return to the origin of the peaks observed at both the type 1 and 2 surfaces. The extraordinary narrowness of the features, as well as the a priori condition of strong electronic correlations, likely precludes any conventional band-theory explanation, such as a flatband or other source of a van Hove singularity.

A possible route to an explanation is provided by dynamical mean field theory (DMFT) [41]. Sharp structures at the Hubbard band edges have been found in treatments of both the dimer [42,43] and single-site Hubbard models [44,45]. These seem to explain such aspects as the lifetime for double occupancy at the quasiparticle peak (analogous to $\tau_{0}$ ) [46] and the asymmetry between features observed in the UHB and the lower Hubbard band (LHB) $[43,45]$. However, the dimer model is not applicable to the type 2 (undimerized) surface, and 
though the single-site model is, the existence of quasiparticles in that model has yet to be firmly established.

The salience of $e$-ph coupling in the real material (absent in DMFT treatments mentioned above) suggests an alternative, intuitive interpretation for the narrow peaks as polaronic bound states. Excess electrons injected into a Mott insulator (doublons) can in principle interact with other excitations to form bound states (see, for example, Ref. [47]). In this case, the charge of the additional electron may be partially screened by the ionic displacement associated with lattice distortions (phonons), reducing the on-site repulsion $U$ and allowing the bound state to split off from the UHB. This interpretation is supported by the fact that the lowest-lying peak at the type 2 surface appears to reside outside the UHB, rather than within it.

As a final observation, in contrast to the clear and abrupt onset of the UHB, the soft and poorly defined onset of occupied states at each type of surface [seen in Fig. 1(d)], which likely results from the hole-doped character of the sample, may explain the apparent absence of narrow structures at the LHB edge, which would correspond to creation of holons by tunneling of electrons from the sample to the tip, and their respective coupling with the amplitude mode. Markers of such interactions, if they exist, may be smeared out or small enough that they are concealed by noise.

In summary, we have observed dynamics induced by electron tunneling into the UHB of Mott insulating $1 T-\mathrm{TaS}_{2}$ surfaces. This reveals unusual, narrow spectral features possibly corresponding to long-lived quasiparticles or bound states, which couple to the amplitude mode of the $\mathrm{CDW}$, the precursor to the Mott state. These observations provide a nanoscale microscopic view of nonequilibrium behavior, complementary to those observations previously provided using ultrafast optical and time-resolved photoemission spectroscopy techniques. Furthermore, they lay down a challenge for theories supporting quantitative model calculations to understand the origin of the unusual UHB edge features, which we anticipate will yield as-yet unknown physics of Mott-Hubbard systems more generally.

\section{MATERIALS AND METHODS}

Crystals of $1 T-\mathrm{TaS}_{2}$ were synthesized and prepared by cleaving at $\approx 77 \mathrm{~K}$ in $\mathrm{UHV}\left(P \sim 10^{-10}\right.$ Torr $)$ as described previously $[5,48]$, before insertion into a modified Unisoku 1300 low-temperature STM system held at $1.5 \mathrm{~K}$ [49]. STM measurements were performed using electrochemically etched tungsten tips, which were characterized and fine-tuned using field ion microscopy and mild indentation at a clean $\mathrm{Cu}(111)$ surface. $d I / d V$ conductance was measured using the lock-in technique with frequency $f_{\text {mod }}=617.3 \mathrm{~Hz}$ and bias modulation of amplitude $V_{\text {mod }}=1 \mathrm{mV}$.
The data that support the findings presented here are available from the corresponding authors upon reasonable request.

\section{ACKNOWLEDGMENTS}

We are grateful to Y. Kohsaka, T. Machida, P. A. Lee, D. Mihailovic, Y. Nomura, N. Nagaosa, M. Civelli, V. Dobrosavljević, and M. J. Rozenberg for helpful discussions. C. J. B. acknowledges support from RIKEN's Programs for Junior Scientists. This work was supported in part by JSPS KAKENHI Grants No. JP18K13511, No. JP19H00653, No. JP19H01855, and No. JP19H05602.

C. J. B, T. H., and Y.I. conceived the project, and M. Y. and Y. I. synthesized the $1 T-\mathrm{TaS}_{2}$ crystals. C. J. B. performed the STM measurements, interpreted the data, and prepared the manuscript with input from all authors.

[1] J. A. Wilson, F. J. Di Salvo, and S. Mahajan, ChargeDensity Waves and Superlattices in the Metallic Layered Transition Metal Dichalcogenides, Adv. Phys. 24, 117 (1975).

[2] M. D. Johannes and I. I. Mazin, Fermi Surface Nesting and the Origin of Charge Density Waves in Metals, Phys. Rev. B 77, 165135 (2008).

[3] J. Bardeen, L. N. Cooper, and J. R. Schrieffer, Theory of Superconductivity, Phys. Rev. 108, 1175 (1957).

[4] G. M. Eliashberg, Interactions between Electrons and Lattice Vibrations in a Superconductor, J. Exp. Theor. Phys. 11, 696 (1960).

[5] C. J. Butler, M. Yoshida, T. Hanaguri, and Y. Iwasa, Mottness versus Unit-Cell Doubling as the Driver of the Insulating State in 1T-TaS 2 , Nat. Commun. 11, 2477 (2020).

[6] P. Fazekas and E. Tosatti, Electrical, Structural and Magnetic Properties of Pure and Doped $1 T-\mathrm{TaS}_{2}$, Philos. Mag. B 39, 229 (1979).

[7] P. Fazekas and E. Tosatti, Charge Carrier Localization in Pure and Doped $1 T-\mathrm{TaS}_{2}$, Physica (Amsterdam) 99B+C, 183 (1980).

[8] L. Perfetti, P. A. Loukakos, M. Lisowski, U. Bovensiepen, M. Wolf, H. Berger, S. Biermann, and A. Georges, Femtosecond Dynamics of Electronic States in the Mott Insulator $1 T-\mathrm{TaS}_{2}$ by Time Resolved Photoelectron Spectroscopy, New J. Phys. 10, 053019 (2008).

[9] A. Mann, E. Baldini, A. Odeh, A. Magrez, H. Berger, and F. Carbone, Probing the Coupling between a Doublon Excitation and the Charge-Density Wave in $\mathrm{TaS}_{2}$ by Ultrafast Optical Spectroscopy, Phys. Rev. B 94, 115122 (2016).

[10] M. Ligges, I. Avigo, D. Golež, H. U. R. Strand, Y. Beyazit, K. Hanff, F. Diekmann, L. Stojchevska, M. Kalläne, P. Zhou, K. Rossnagel, M. Eckstein, P. Werner, and U. Bovensiepen, Ultrafast Doublon Dynamics in Photoexcited $1 T-\mathrm{TaS}_{2}$, Phys. Rev. Lett. 120, 166401 (2018).

[11] J. C. Petersen, S. Kaiser, N. Dean, A. Simoncig, H. Y. Liu, A. L. Cavalieri, C. Cacho, I. C. E. Turcu, E. Springate, F. Frassetto, L. Poletto, S. S. Dhesi, H. Berger, and A. Cavalleri, Clocking the Melting Transition of Charge 
and Lattice Order in $1 T-\mathrm{TaS}_{2}$ with Ultrafast ExtremeUltraviolet Angle-Resolved Photoemission Spectroscopy, Phys. Rev. Lett. 107, 177402 (2011).

[12] S. Hellmann, T. Rohwer, M. Kalläne, K. Hanff, C. Sohrt, A. Stange, A. Carr, M. M. Murnane, H. C. Kapteyn, L. Kipp, M. Bauer, and K. Rossnagel, Time-Domain Classification of Charge-Density-Wave Insulators, Nat. Commun. 3, 1069 (2012).

[13] J. Zhang, C. Lian, M. Guan, W. Ma, H. Fu, H. Guo, and S. Meng, Photoexcitation Induced Quantum Dynamics of Charge Density Wave and Emergence of a Collective Mode in 1T-TaS 2 , Nano Lett. 19, 6027 (2019).

[14] I. Avigo, F. Queisser, P. Zhou, M. Ligges, K. Rossnagel, R. Schützhold, and U. Bovensiepen, Doublon Bottleneck in the Ultrafast Relaxation Dynamics of Hot Electrons in $1 T-\mathrm{TaS}_{2}$, Phys. Rev. Research 2, 022046(R) (2020).

[15] T. Ritschel, H. Berger, and J. Geck, Stacking-Driven Gap Formation in Layered $1 T-\mathrm{TaS}_{2}$, Phys. Rev. B 98, 195134 (2018).

[16] S.-H. Lee, J. S. Goh, and D. Cho, Origin of the Insulating Phase and First-Order Metal-Insulator Transition in $1 T$-TaS 2 , Phys. Rev. Lett. 122, 106404 (2019).

[17] S. Tanda, T. Sambongi, T. Tani, and S. Tanaka, X-Ray Study of Charge Density Wave Structure of $1 T-\mathrm{TaS}_{2}$, J. Phys. Soc. Jpn. 53, 476 (1984).

[18] M. Naito, H. Nishihara, and S. Tanaka, Nuclear Quadrupole Resonance in the Charge Density Wave State of $1 T-\mathrm{TaS}_{2}$, J. Phys. Soc. Jpn. 53, 1610-1613 (1984).

[19] M. Naito, H. Nishihara, and S. Tanaka, Nuclear Magnetic Resonance and Nuclear Quadrupole Resonance Study of ${ }^{181} \mathrm{Ta}$ in the Commensurate Charge Density Wave State of $1 T-\mathrm{TaS}_{2}$, J. Phys. Soc. Jpn. 55, 2410 (1986).

[20] Q. Stahl, M. Kusch, F. Heinsch, G. Garbarino, N. Kretzschmar, K. Hanff, K. Rossnagel, J. Geck, and T. Ritschel, Collapse of Layer Dimerization in the Photo-Induced Hidden State of $1 T-\mathrm{TaS}_{2}$, Nat. Commun. 11, 1247 (2020).

[21] L. Le Guyader, T. Chase, A. H. Reid, R. K. Li, D. Svetin, X. Shen, T. Vecchione, X. J. Wang, D. Mihailovic, and H. A. Dür, Stacking Order Dynamics in the Quasi-Two-Dimensional Dichalcogenide $1 T-\mathrm{TaS}_{2}$ Probed with MeV Ultrafast Electron Diffraction, Struct. Dyn. 4, 044020 (2017).

[22] G. von Witte, T. Kißlinger, J. G. Horstmann, K. Rossnagel, M. A. Schneider, C. Ropers, and L. Hammer, Surface Structure and Stacking of the Commensurate $(\sqrt{13} \times \sqrt{13}) R 13.9^{\circ}$ Charge Density Wave Phase of $1 T-\mathrm{TaS}_{2}(0001)$, Phys. Rev. B 100, 155407 (2019).

[23] Y. D. Wang, W. L. Yao, Z. M. Xin, T. T. Han, Z. G. Wang, L. Chen, C. Cai, Y. Li, and Y. Zhang, Band Insulator to Mott Insulator Transition in 1T-TaS 2 , Nat. Commun. 11, 4215 (2020).

[24] A. Fuhrmann, D. Heilmann, and H. Monien, From Mott Insulator to Band Insulator: A Dynamical Mean-Field Theory Study, Phys. Rev. B 73, 245118 (2006).

[25] K. Bu, W. Zhang, Y. Fei, Z. Wu, Y. Zheng, J. Gao, X. Luo, Y.-P. Sun, and Y. Yin, Possible Strain Induced Mott Gap Collapse in 1T-TaS 2 , Commun. Phys. 2, 146 (2019).

[26] See Supplemental Material at http://link.aps.org/ supplemental/10.1103/PhysRevX.11.011059 for a detailed discussion of setpoint-related and tip-induced effects, a comparison of conductance spectra acquired at type 1 and type 2 surfaces, a demonstration of the reproducibility of the observation of conductance peaks, spectroscopic imaging near defects, and further discussion of the observed phonon energy.

[27] K. Ishizaka, R. Eguchi, S. Tsuda, A. Chainani, T. Yokoya, T. Kiss, T. Shimojima, T. Togashi, S. Watanabe, C.-T. Chen, Y. Takano, M. Nagao, I. Sakaguchi, T. Takenouchi, H. Kawarada, and S. Shin, Temperature-Dependent Localized Excitations of Doped Carriers in Superconducting Diamond, Phys. Rev. Lett. 100, 166402 (2008).

[28] S. W. Wu, G. V. Nazin, X. Chen, X. H. Qiu, and W. Ho, Control of Relative Tunneling Rates in Single Molecule Bipolar Electron Transport, Phys. Rev. Lett. 93, 236802 (2004).

[29] X. H. Qiu, G. V. Nazin, and W. Ho, Vibronic States in Single Molecule Electron Transport, Phys. Rev. Lett. 92, 206102 (2004).

[30] G. V. Nazin, S. W. Wu, and W. Ho, Tunneling Rates in Electron Transport through Double-Barrier Molecular Junctions in a Scanning Tunneling Microscope, Proc. Natl. Acad. Sci. U.S.A. 102, 8832 (2005).

[31] A. Tokmakoff, Introductory Quantum Mechanics II, Spring 2009, Massachusetts Institute of Technology, Cambridge, MA, MIT OpenCourseWare, https://ocw.mit.edu/courses/ chemistry/5-74-introductory-quantum-mechanics-ii-spring2009/lecture-notes/MIT5_74s09_lec08.pdf, License: Creative Commons BY-NC-SA.

[32] A. M. Stoneham, J. Gavartin, A. L. Shluger, A. V. Kimmel, D. Muñoz Ramo, H. M. Rønnow, G. Aeppli, and C. Renner, Trapping, Self-Trapping and the Polaron Family, J. Phys. Condens. Matter 19, 255208 (2007).

[33] M. Hangyo, S.-I. Nakashima, and A. Mitsuishi, Raman Spectroscopic studies of MX2-Type Layered Compounds, Ferroelectrics 52, 151 (1983).

[34] O. R. Albertini, R. Zhao, R. L. McCann, S. Feng, M. Terrones, J. K. Freericks, J. A. Robinson, and A. Y. Liu, Zone-Center Phonons of Bulk, Few-Layer, and Monolayer $1 T-\mathrm{TaS}_{2}$ : Detection of Commensurate Charge Density Wave Phase through Raman Scattering, Phys. Rev. B 93, 214109 (2016).

[35] P. Kusar, T. Mertelj, V. V. Kabanov, J.-H. Chu, I. R. Fisher, H. Berger, L. Forró, and D. Mihailovic, Anharmonic OrderParameter Oscillations and Lattice Coupling in Strongly Driven $1 T-\mathrm{TaS}_{2}$ and $\mathrm{TbTe}_{3}$ Charge-Density-Wave Compounds: A Multiple-Pulse Femtosecond Laser Spectroscopy Study, Phys. Rev. B 83, 035104 (2011).

[36] L. Stojchevska, I. Vaskivskyi, T. Mertelj, P. Kusar, D. Svetin, S. Brazovskii, and D. Mihailovic, Ultrafast Switching to a Stable Hidden Quantum State in an Electronic Crystal, Science 344, 177 (2014).

[37] Y. Toda, K. Tateishi, and S. Tanda, Anomalous Coherent Phonon Oscillations in the Commensurate Phase of the Quasi-Two-Dimensional $1 T-\mathrm{TaS}_{2}$ Compound, Phys. Rev. B 70, 033106 (2004).

[38] N. Dean, J. C. Petersen, D. Fausti, R. I. Tobey, S. Kaiser, L. V. Gasparov, H. Berger, and A. Cavalleri, Polaronic Conductivity in the Photoinduced Phase of $1 T-\mathrm{TaS}_{2}$, Phys. Rev. Lett. 106, 016401 (2011).

[39] Q. Hu, C. Yin, L. Zhang, L. Lei, Z. Wang, Z. Chen, J. Tang, and R. Ang, Direct Observation of Melted Mott State 
Evidenced from Raman Scattering in $1 T-\mathrm{TaS}_{2}$ Single Crystal, Chin. Phys. B 27, 017104 (2018).

[40] R. Sensarma, D. Pekker, E. Altman, E. Demler, N. Strohmaier, D. Greif, R. Jördens, L. Tarruell, H. Moritz, and T. Esslinger, Lifetime of Double Occupancies in the Fermi-Hubbard Model, Phys. Rev. B 82, 224302 (2010).

[41] A. Georges, G. Kotliar, W. Krauth, and M. J. Rozenberg, Dynamical Mean-Field Theory of Strongly Correlated Fermion Systems and the Limit of Infinite Dimensions, Rev. Mod. Phys. 68, 13 (1996).

[42] O. Nájera, M. Civelli, V. Dobrosavljević, and M. J. Rozenberg, Resolving the $\mathrm{VO}_{2}$ Controversy: Mott Mechanism Dominates the Insulator-to-Metal Transition, Phys. Rev. B 95, 035113 (2017).

[43] O. Nájera, M. Civelli, V. Dobrosavljević, and M. J. Rozenberg, Multiple Crossovers and Coherent States in a Mott-Peierls Insulator, Phys. Rev. B 97, 045108 (2018).

[44] S. Nishimoto, F. Gebhard, and E. Jeckelmann, Dynamical Density-Matrix Renormalization Group for the
Mott-Hubbard Insulator in High Dimensions, J. Phys. Condens. Matter 16, 7063 (2004).

[45] M. Granath and J. Schött, Signatures of Coherent Electronic Quasiparticles in the Paramagnetic Mott Insulator, Phys. Rev. B 90, 235129 (2014).

[46] M. Ganahl, M. Aichhorn, H. G. Evertz, P. Thunström, K. Held, and F. Verstraete, Efficient DMFT Impurity Solver Using Real-Time Dynamics with Matrix Product States, Phys. Rev. B 92, 155132 (2015).

[47] T. Terashige, T. Ono, T. Miyamoto, T. Morimoto, H. Yamakawa, N. Kida, T. Ito, T. Sasagawa, T. Tohyama, and H. Okamoto, Doublon-Holon Pairing Mechanism via Exchange Interaction in Two-Dimensional Cuprate Mott Insulators, Sci. Adv. 5, eaav2187 (2019).

[48] T. Tani, K. Okajima, T. Itoh, and S. Tanaka, Electronic Transport Properties in $1 T-\mathrm{TaS}_{2}$, Physica (Amsterdam) 105B+C, 127 (1981).

[49] T. Hanaguri, Development of High-Field STM and Its Application to the Study on Magnetically Tuned Criticality in $\mathrm{Sr}_{3} \mathrm{Ru}_{2} \mathrm{O}_{7}$, J. Phys. Conf. Ser. 51, 514 (2006). 